\section{On the transferability of tissue engineering technologies to the design of tissue models}

\author{
Gianluca Ciardelli \\ Department of Mechanical and \\ Aerospace Engineering (DIMEAS), \\ Politecnico di Torino, Turin, Italy
}

\begin{abstract}
3D tissue-engineered models are promising tools in the screening and evaluation of drugs and therapies as well as in the investigation of the molecular mechanisms involved in disease onset and progression. In this context, we describe our efforts in soft tissue replication, to design in vitro models that have the potential to provide better insight into the development of ageing process and related pathologies, with particular reference to the cardiovascular field.
\end{abstract}

\section{Introduction}

3D tissue-engineered models are promising tools in the screening and evaluation of drugs and therapies as well as in the investigation of the molecular mechanisms involved in disease onset and progression.

In a $3 \mathrm{D}$ bioengineered model, the scaffold should finely reproduce the architecture and composition of the native tissue, in order to properly regulate cell behaviour, such as spreading, proliferation, differentiation, and extracellular matrix (ECM) production, alike to what they do in vivo. In order to successfully mimic a tissue, several aspects must be considered when designing a tissue model. Among others, cell sources and 3D scaffolds architecture and composition (both in bulk and on the surface) have been investigated in depth (Figure 1).

The biomaterial choice is a crucial element, since it strongly affects cellular functions. It should be based on the material mechanical properties, as the one of the scaffolds should match those of the tissue to be modelled, as thoroughly highlighted by mechanobiology studies. ${ }^{1}$ In the replication of hard tissues, due to their high stiffness and load- bearing properties, ceramics and their composites are generally used, while natural and synthetic polymers are appropriate choices in the engineering of soft tissues. ${ }^{2}$

In soft tissue replication, in vitro models of heart tissue have the potential to provide better insight into the mechanisms of heart diseases, which are the leading cause of mortality in the world. Moreover, myocardial models could be a useful tool in the evaluation of drugs cardiotoxicity. In cardiac tissue engineering, the most investigated natural polymers are collagen, gelatin, fibrin, and alginate, and among synthetic polymers, degradable polyesters and their copolymers have been mainly explored. For instance, since the myocardial tissue has to bear the contractile and expansive forces produced at each cardiac cycle, elastomeric polymers, such as poly(glycerol sebacate) and polyurethanes have been mainly studied.

\section{Results}

Our group extensively worked in the synthesis of biodegradable polyurethanes for contractile tissues applications. In a previous article, we identified a promising polyester-urethane (PUR), showing low Young's modulus and elastomeric behaviour. ${ }^{3}$ This PUR was synthesized from poly( $\varepsilon$-caprolactone) diol, 1,4-butanediisocyanate and L-lysine ethyl ester. In a succeeding article, this PUR was used to obtain porous scaffolds by thermally induced phase separation (TIPS). The scaffolds were functionalized with fibronectin by plasma treatment, and seeded with primary rat neonatal cardiomyocytes. ${ }^{4}$ Morphological and mechanical analysis exhibited a porealigned scaffold formation, with mechanical properties and architecture resembling the one of the myocardium. Cardiomyocyte viability was stable for 14 days of experimentation, comprising beating activity and cell adhesion. The improved levels of phosphorylation of AKT and ERK1/2 elucidated the prolonged cell survival. The variation of cardiac muscle and glucose genes indicated a transition phase in cardiomyocyte maturation, with an early evolution to an adult phenotype and a permanence of some fetal characteristics. These results represented a first step towards the development of a new generation of myocardial models. A further step involved the fabrication of scaffolds with improved controlled geometry, which can be obtained via rapid prototyping (RP) procedures.In detail, the PUR was microfabricated into $0^{\circ} / 90^{\circ}$ square-grid multilayered scaffolds through a custom-made melt-extrusion additive manufacturing instrument and surface functionalized with laminin-1 (cardiac niche ECM component, sample acronym PUR-LN1) or gelatin (low-cost adhesion protein, sample acronym PUR-G) through plasma treatment followed by carbodiimide chemistry. 5 PUR-
Correspondence: Gianluca Ciardelli, Department of Mechanical and Aerospace Engineering (DIMEAS), Politecnico di Torino, Turin, Italy.

E-mail: gianluca.ciardelli@polito.it

Key words: Polyurethanes; bioengineered models; soft tissues; thermoplastic polymers; hydrogels.

Conference presentation: this paper was presented at the Second Centro 3R Annual Meeting - 3Rs in Italian Universities, 2019, June 20-21, University of Genoa, Italy.

Received for publication: 28 October 2019. Accepted for publication: 6 November 2019 .

This work is licensed under a Creative Commons Attribution NonCommercial 4.0 License (CC BY-NC 4.0).

CC Copyright: the Author(s), 2019

Licensee PAGEPress, Italy

Biomedical Science and Engineering 2019; 3(s2):85 doi:10.4081/bse.2019.85

G and PUR-LN1 scaffolds both improved cardiac progenitor cell (CPC) adhesion, but LN1 functionalization turned out to be superior in promoting CPC proliferation, protection from apoptosis and expression of differentiation markers for cardiomyocytes, endothelial and smooth muscle cells. PURLN1 subcutaneously implanted in mice evoked a weak inflammation and integrated with the host surrounding tissue, evidencing a significant blood vessel density around the scaffolds. In a different approach, thermo- and photo-curable bioinks were designed for the bioprinting of cellularized constructs to be used for reparative/regenerative purposes or as in vitro bioengineered models of soft tissues. The thermo-sensitive nature of the developed bioinks allowed an easy and homogeneous cell dispersion, through the addition of the cells to the hydrogels at a temperature lower than their gelation temperature (below $25^{\circ} \mathrm{C}$ ), when the systems were in the sol or semi-gel phase. After loading in the syringe, cellularized hydrogels were subjected to a fast solto-gel transition with increasing temperature. Syringe equilibrium temperature and, as a consequence, bioink viscosity were finely optimized to maximize the outcomes of the printing process, while minimizing cell suffering due to applied shear stress. To this aim, the possibility to print the developed hydrogels as biphasic sol/gel systems or fully developed gels able to keep their shape upon printing was exploited to finely balance the two concurrent needs of high resolution and poor cell suffering and death. 


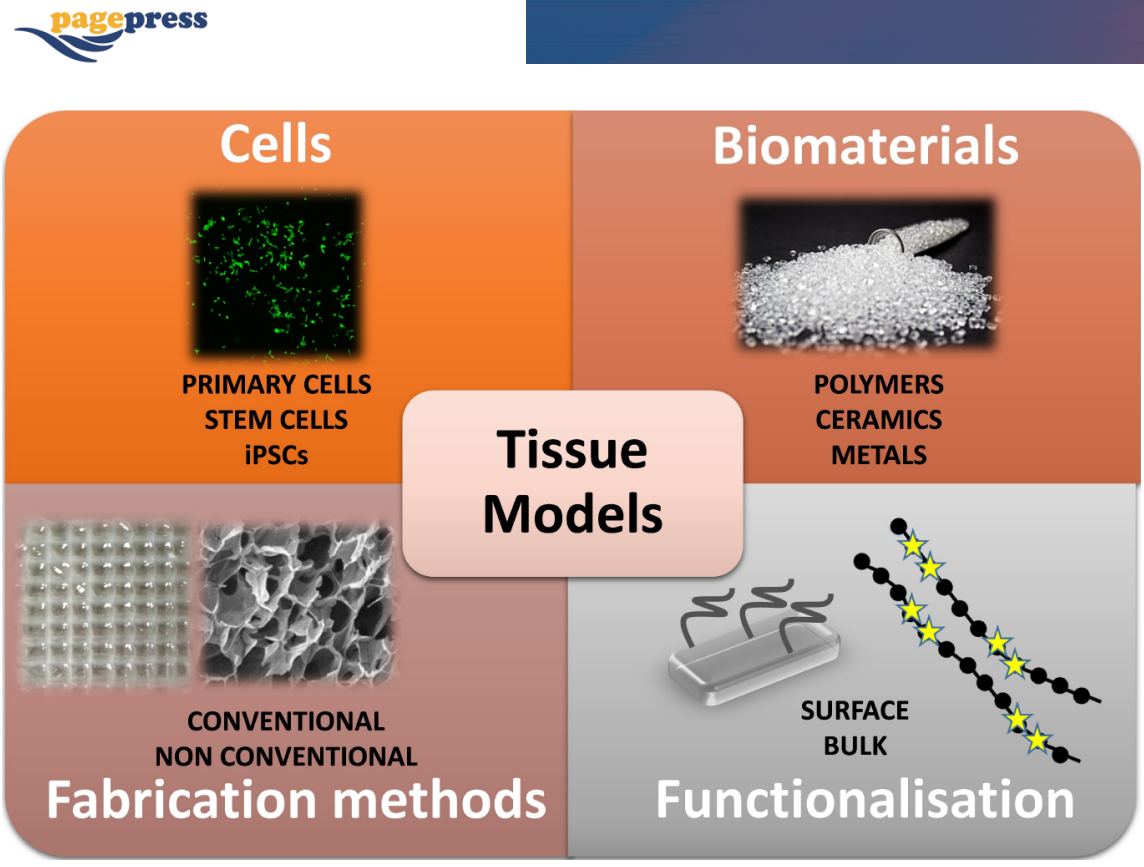

Figure 1. TE approach for the development of in vitro models.

Moreover, the fast sol-to-gel transition of the developed systems ensured a homogeneous cell distribution, avoiding cell sedimentation during the printing process. Eventually, a further degree of freedom was provided to the developed bioinks by making them photo-responsive, allowing a fine modulation of the final mechanical properties and stability in aqueous environment of the scaffolds. By working on bioink compo- sition, multilayered scaffolds with residence time in aqueous environment ranging between few weeks up to more than 2 months were designed. Additionally, gel stiffness was modulated within the range 1$100 \mathrm{kPa}$, thus making the developed bioinks suitable for a wide variety of applications in the biomedical field, such as in brain, skin, liver, kidney and muscle tissue engineering.

\section{References}

1. Chan BP, Leong KW. Scaffolding in tissue engineering: General approaches and tissue-specific considerations. Eur Spine J 2008;17:467-79.

2. Seidi A, Ramalingam M, ElloumiHannachi I, et al. Gradient biomaterials for soft-to-hard interface tissue engineering. Acta Biomater 2011;7:144151.

3. Sartori S, Boffito M, Serafini P, et al. Synthesis and structure-property relationship of polyester-urethanes and their evaluation for the regeneration of contractile tissues. React Funct Polym 2013;73:1366-76.

4. Vozzi F, Logrand F, Cabiati M, et al. Biomimetic engineering of the cardiac tissue through processing, functionalization, and biological characterization of polyester urethanes. Biomed Mater 2018;13:055006.

5. Boffito M, Di Meglio F, Mozetic P, et al. Surface functionalization of polyurethane scaffolds mimicking the myocardial microenvironment to support cardiac primitive cells. PLoS One 2018;13:e0199896. 